\title{
Spatially modulated magnetic structures in thin films
}

\author{
W Selke§, D Catrein§, and M Pleimling† \\ $\S$ Institut für Theoretische Physik, Technische Hochschule, D-52056 Aachen, \\ Germany \\ † Institut für Theoretische Physik 1, Universität Erlangen-Nürnberg, D-91058 \\ Erlangen, Germany \\ ¥ Laboratoire de Physique des Matériaux Université Henri Poincaré Nancy I, B.P. \\ 239, F-54506 Vandœuvre lès Nancy Cedex, France
}

\begin{abstract}
The axial next-nearest neighbour Ising (ANNNI) model is studied for thin films of up to $L=10$ layers, with a distinct phase diagram for each film thickness. The systematics of the ordered phases, as obtained from mean-field theory, Monte Carlo simulations, and low temperature expansions, is discussed. Results are compared to those for the ANNNI model in the limit $L \longrightarrow \infty$.
\end{abstract}

PACS numbers: 68.35.Rh, 75.70.Mk, 64.70.Rh 
In recent years magnetism in thin films of a few atomic layers has attracted much interest [1]. However, studies on the influence of the layer thickness, $L$, on spatially modulated magnetic structures seem to be very scarce [2].

In the following, we shall deal with this aspect by analysing phase diagrams of the axial next-nearest neighbour Ising (ANNNI) model [3, 4, 5] on a simple cubic lattice (setting the lattice constant equal to one) consisting of rather few, but large layers. The competing interactions are supposed to be ferromagnetic between neighbouring spins in each layer, $J_{0}>0$, as well as between neighbouring spins in adjacent layers, $J_{1}>0$, and to be antiferromagnetic, $J_{2}<0$, between axial next-nearest neighbour spins, distinguishing one of the three cubic axes, say, the $z$-axis. The resulting magnetic structures may exhibit non-trivial spatial modulations along the $z$-axis. In the case of indefinitely many and indefinitely large layers, the model displays a phase diagram with a plenitude of commensurate phases, including those springing from the multiphase point at zero temperature and those emerging from structure combination branching processes at finite temperatures, as well as incommensurate structures, see the reviews [3, 4, 5].

Here we shall consider thin films of $L$ layers, with free boundary conditions at the surface layers. Note that the ANNNI model on finite lattices, connecting the first and last layers by periodic boundary conditions, has been studied quite extensively before, attempting to predict properties for $L \longrightarrow \infty$. The scope of the present study is, however, to determine the phase diagram of the ANNNI model with free boundary conditions at fixed film thickness. Attention may be also drawn to recent work on the semi-infinite ANNNI model, analysing critical surface properties near the Lifshitz point [6], which partly motivated our investigation.

To establish the phase diagrams for thin films, in particular for $L=3,4, \ldots, 10$, we used mean-field theory, Monte Carlo simulations and low temperature expansions. For simplicity, we set $J_{0}=J_{1}$, both in the surface layers and in the bulk of the film. In mean-field approximation [7, 8, 9], we solved the set of coupled non-linear equations for the layer magnetizations, $m(z=1, \ldots, L)$, by standard iteration procedures starting from all possible combinations of fully ordered, $m=1$ or $m=-1$, or completely disordered, $m=0$, layers, i. e. from, in principle, $3^{L}$ distinct configurations. To identify the stable phase, at given temperature, $\tau=k_{B} T / J_{0}$, and ratio of competing interactions, $\kappa=-J_{2} / J_{1}$, we calculated the free energy for all resulting structures. This approach seems to be suitable to map correctly, in mean-field approximation, the entire phase diagram. The Monte Carlo simulations, using the single-spin flip algorithm, were performed for lattices with $K \times K$ spins in each layer connected by periodic boundary conditions (for thicker films, an efficient layer flip algorithm may be useful [10]). To circumvent finite size effects due to the layer size, $K$ has to be chosen sufficiently large. Actually, we varied $K$ from 10 to 100. To identify the phases springing directly from the degenerate ground states at $\kappa=1 / 2$, low temperature expansions to leading, first order [11, 12] were found to suffice in most cases.

At zero temperature, both for $L$ odd and even, the ferromagnetic state is stable 
at $\kappa<1 / 2$. For $L$ even, at $\kappa>1 / 2$, the ground state is the $\left\langle 2^{L / 2}\right\rangle$ structure, where two layers of "up" ("down") spins are followed by two layers of "down" ("up") spins, with totally $(L / 2)$ pairs of such layers (in general, $\langle\ldots k l \ldots\rangle$ refers to a phase at low temperatures where a " $k$-band", i. e. spins in $k$ adjacent layers being oriented (predominantly) in one direction, say, "+", is followed by a $l$-band of spins being oriented (predominantly) in the opposite direction, say, "-"12 ). For $L$ odd, structures consisting of 2 -bands and one 3 -band have the lowest energy at $1 / 2<\kappa<1$, while at $\kappa>1$ the ground state contains 2 -bands with a 1 -band at one of the surfaces (of course, $\kappa=1$ plays no special role for the ANNNI model with $L \longrightarrow \infty$ ). The transition line, at $\kappa \approx 1$, between the corresponding phases is, at low temperatures, of first order.

The degeneracy, $D_{L}$, of the multiphase point, at $(\kappa=1 / 2, \tau=0)$, is related to the Fibonacci sequence by $D_{L}=D_{L-2}+D_{L-1}$, with $D_{2}=D_{3}=2$. We studied the vicinity of that point by using low temperature expansions [11, [1]. In the limit $L \longrightarrow \infty$ [11], indefinitely many periodic, commensurate phases spring directly from that point, namely the ferromagnetic phase and phases in each of which 3-bands are separated by a fixed number, $j$, of 2 -bands, $j=0,1,2, \ldots \infty$. In thin films, obviously only a few phases may arise from the multiphase point, in particular, the ferromagnetic phase as well as additional phases formed mostly by 2 -bands and 3 -bands. For $L$ even, the $\left\langle 2^{L / 2}\right\rangle$ phase will be among the additional phases, while for $L$ odd, all of these phases will have at least one 3-band (when it is just one 3-band, then that band will be preferably in the center of the film). Note that structures consisting of 3-bands and a supplementary single 4 -band or a single 5 -band at one of the surfaces of the film occur when $L=3 n+1$ or $L=3 n+2, n=2,3, \ldots$ Examples of those phases bordering, at low temperatures, the ferromagnetic phase are the $\langle 43\rangle,\langle 53\rangle$, and $\langle 433\rangle$ phases. Again in contrast to the case $L \longrightarrow \infty$, phases with 2 -bands and pairs of 3 -bands may be stable as well; examples are the $\langle 2332\rangle$ and $\langle 332\rangle$ phases.- To understand the phase diagram in the vicinity of the multiphase point one should be aware of the fact that the stable phases correspond to the ground state structures with a low energy and a large number of lowest-energy excitations. The latter property favours 3-bands, where the spins in both edge layers can be excited easily when both the preceeding and the following band comprise at least two layers.

Examples of entire phase diagrams are depicted in Figs. 1 and 2 (a more complete set of phase diagrams can be found elsewhere [13]). In general, for thin films, there are not many ordered phases. The structures close to the transition to the paramagnetic phase, $T_{c}$, may be obtained in mean-field approximation without difficulty from the linearized theory. One finds that the pattern of the layer magnetization $m(z)$ is either symmetric or antisymmetric about the center of the film. Accordingly, some of the low temperature phases discussed above cannot extend up to $T_{c}$, e. g. the $\langle 43\rangle$ phase (note that mean-field theory seems to reproduce the low temperature behaviour qualitatively correctly). Each of the symmetric or antisymmetric phases is usually stable in a rather wide region of the phase diagram, for the thin films we considered. These features, symmetrization and possible instability of low temperature phases close to $T_{c}$, are in 

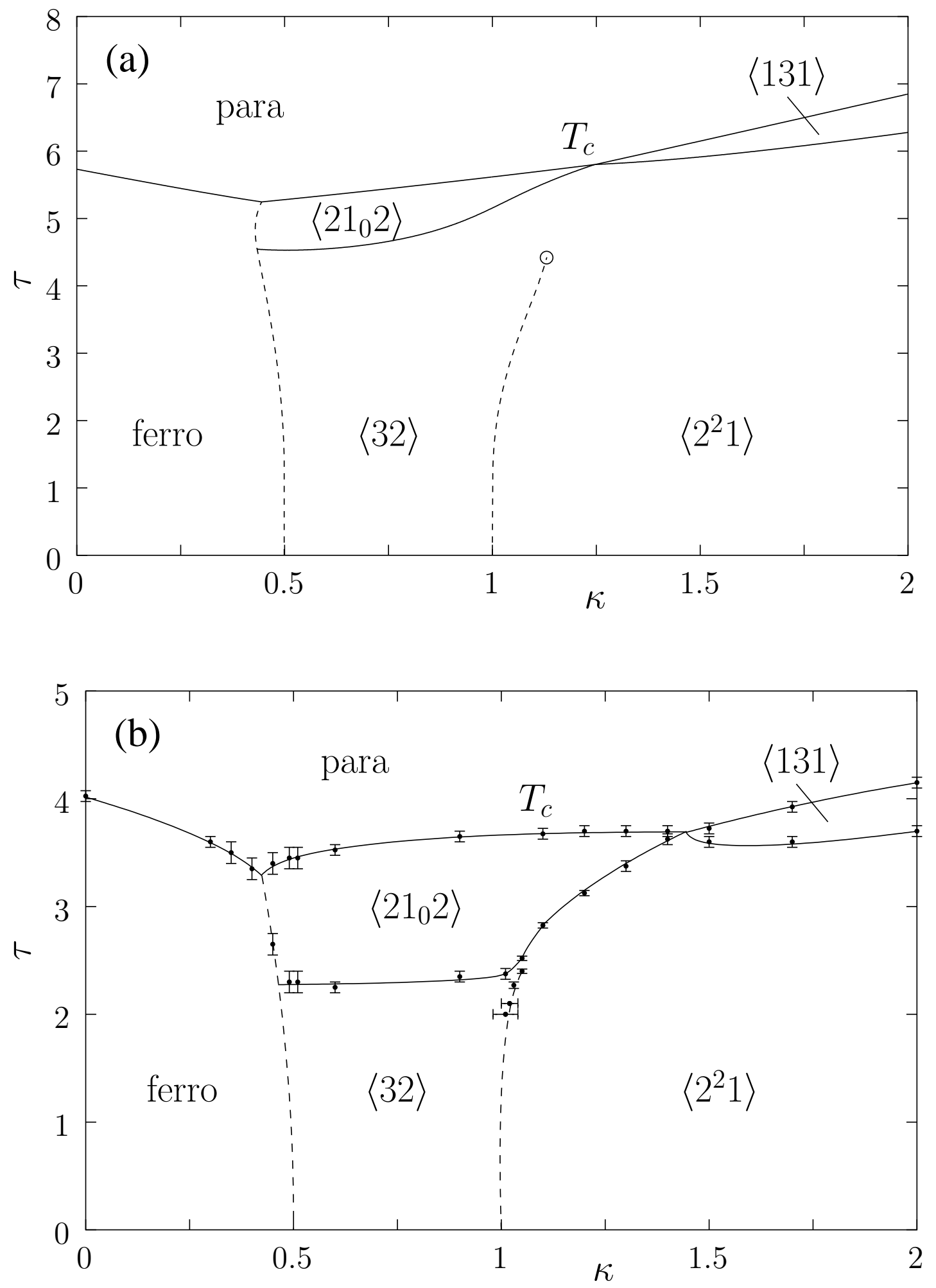

Figure 1. Phase diagram of the ANNNI model for $L=5$ in the $\left(\tau=k_{B} T / J_{0}, \kappa=\right.$ $\left.-J_{2} / J_{1}\right)$-plane using (a) mean-field theory and (b) Monte Carlo simulations. Dashed (solid) lines denote transition lines of first (second) order. In the Monte Carlo case, the lines are guides to the eye. 
Modulated magnetic structures
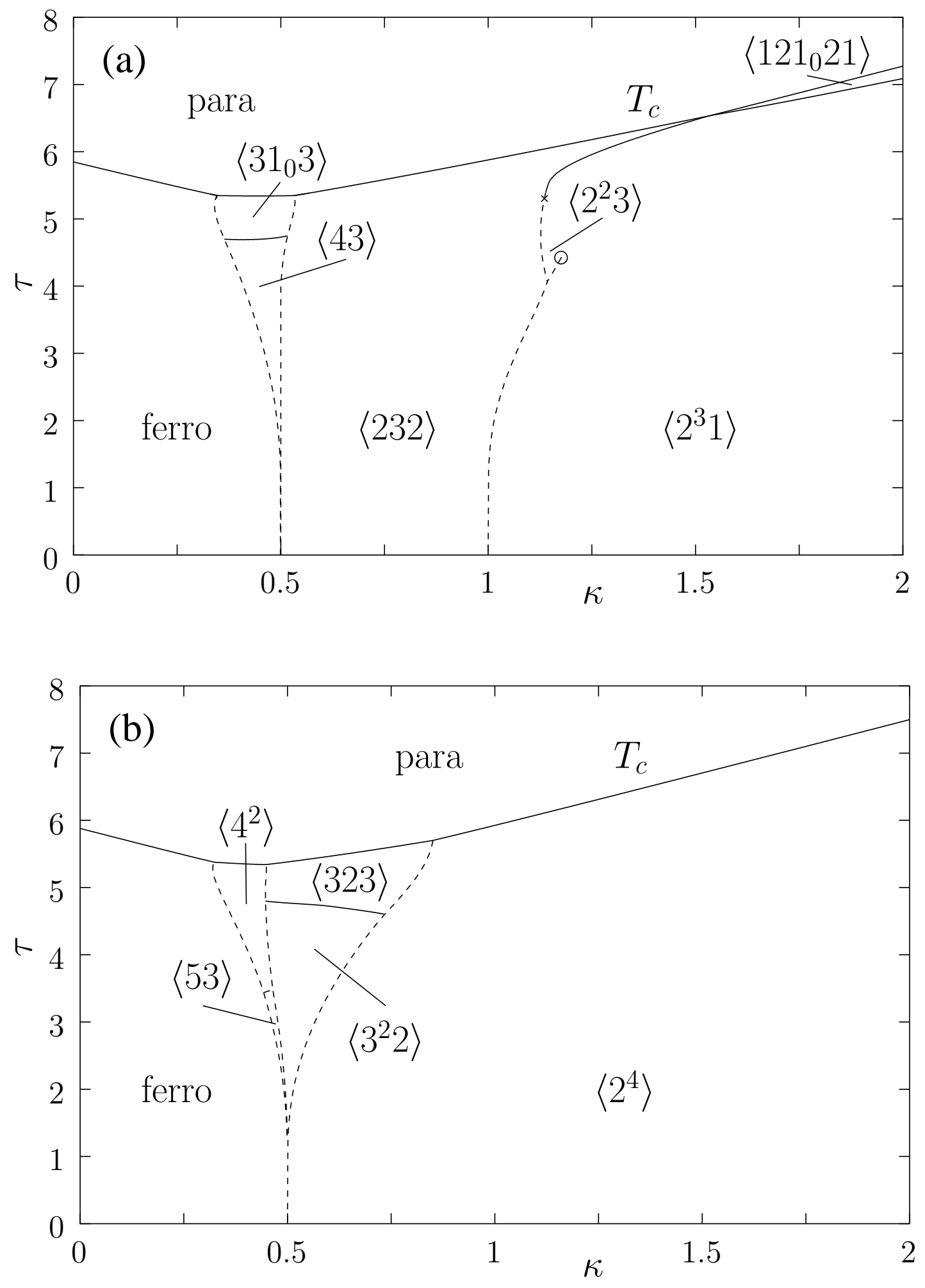

Figure 2. Phase diagrams of the ANNNI model, using mean-field theory, for thin films with (a) $L=7$ and (b) $L=8$ layers. 
marked contrast to the mean-field description of the ANNNI model with $L \longrightarrow \infty$, where the structures below $T_{c}$ form a devil's staircase. Perhaps most interestingly, we identified a new type of antisymmetric phase, with the center layer of the film being disordered, for odd $L$. Examples are the $\left\langle 11_{0} 1\right\rangle,\left\langle 21_{0} 2\right\rangle,\left\langle 31_{0} 3\right\rangle$, and $\left\langle 41_{0} 4\right\rangle$ phases for $L=3,5,7$, and 9 (we modified the standard low temperature notation in an obvious way by denoting the disordered layer by " $1_{0}$ ", i. e. for instance, $\left\langle 11_{0} 1\right\rangle$ stands for a magnetization pattern where $m(1)>0, m(2)=0$, and $m(3)<0)$. For $L=9$, at larger ratio of the competing interactions, $\kappa$, one finds another partially disordered phase, namely the $\left\langle 221_{0} 22\right\rangle$ phase.

Partially disordered phases, with period arrangements of disordered layers, occur in mean-field theory of the ANNNI model, $L \longrightarrow \infty$, at sufficiently weak intra-plane couplings, $J_{0}$ [14, 15]. They turned out to be an artefact of the approximation, as seen in Monte Carlo simulations [16]. However, in the present case, simulations confirm the prediction of mean-field theory of disordered center layers, as we checked for $L$ up to 9. Obviously, those phases are expected to exist only above the critical temperature of the two-dimensional Ising model, see Fig. 1.

Note that the sign of the magnetization $m(z)$ may change, at higher temperatures, in some layers without any phase transition. To characterise such a phase, we based the notation, used in the phase diagrams, Figs. 1 and 2, on the magnetization pattern of that phase in the low temperature region. An example is the $\langle 323\rangle$ phase, see Fig. 2, where, close to $T_{c}$, the magnetization in the third and sixth layers may change sign so that the 2-band in the center expands, formally, to a 4-band, with the 3-bands shrinking to 2bands. However, the phase keeps its symmetry, and there is no transition or degeneracy associated with this change of sign.

At intermediate temperatures, interesting features are observed as well. In meanfield theory, the transition line to the $\left\langle 2^{(L-1) / 2} 1\right\rangle$ phase at $\kappa \approx 1$, being of first order at low temperatures, ends in a critical point $(L=5,7$, and 9). Simulations, for $L=5$, seem to be in qualitative agreement, see Fig. 1, but careful and extensive finite-size analyses are needed to establish this aspect definitely. Moreover, at intermediate temperatures additional phases may be stabilized, springing neither directly from the ground states nor being (anti-)symmetric. An example is the $\langle 54\rangle$ phase for $L=9$ (undergoing, at higher temperatures, a transition to the symmetric $\left\langle 41_{0} 4\right\rangle$ phase). Indeed, if $L$ gets large enough, structure combination branching processes may happen, when being compatible with the film thickness (again, with, possibly, slight rearrangements and modifications of bands, especially near the surface, as in the low temperature region).

In summary, most of the distinct commensurate phases of the ANNNI model in the limit $L \longrightarrow \infty$ are suppressed in thin films. Perhaps surprisingly, however, modified structures and novel phases are induced by the (small) thickness of the film, L, for example, among the phases springing directly from the multiphase point and among those close to the paramagnetic transition line. Certainly, it may be interesting to study the robustness of the findings against varying model parameters such as, for instance, the intralayer coupling, $J_{0}$, in the surface planes. 


\section{Acknowledgments}

Useful discussions with J. Hager are gratefully acknowledged.

\section{References}

[1] Poulopoulos P and Baberschke K 1999 J. Phys.-Condens. Mat. 119495

[2] Spišák D and Hafner J 1997 Phys. Rev. B56 2646

[3] Selke W 1988 Phys. Rep. 170213

[4] Yeomans JM 1988 Adv. Phys. 41151

[5] Neubert B, Pleimling M, and Siems R 1998 Ferroelectrics 208-209 141

[6] Binder K and Frisch H L 1999 Eur. Phys. J. B10 71

[7] Bak P 1982 Rep. Prog. Phys. 45587

[8] Selke W and Duxbury PM 1984 Z. Phys. B57 49

[9] Janssen T and Tjon J A 1983 J. Phys. A16 673

[10] Sato A and Matsubara F 1999 Phys. Rev. B60 10316

[11] Fisher M E and Selke W 1980 Phys. Rev. Lett. 441502

[12] Fisher M E and Selke W 1981 Phil. Trans. R. Soc. 3021

[13] Catrein D 2000, Diploma thesis, RWTH-Aachen

[14] Yokoi C S O, Coutinho-Filho M D, and Salinas S R 1981 Phys. Rev. B24 4047

[15] Nakanishi K 1992 J. Phys. Soc. Japan 612901

[16] Rotthaus F and Selke W 1993 J. Phys. Soc. Japan 62378 